\title{
Maximally Flat Waveforms with Finite Number of Harmonics in Class- $F$ Power Amplifiers
}

\author{
Anamarija Juhas and Ladislav A. Novak \\ Department of Power, Electronics and Communication Engineering, Faculty of Technical Sciences, University of Novi Sad, Serbia \\ Correspondence should be addressed to Ladislav A. Novak; ladislav@uns.ac.rs
}

Received 1 August 2013; Accepted 21 September 2013

Academic Editor: Xu Zhang

Copyright (C) 2013 A. Juhas and L. A. Novak. This is an open access article distributed under the Creative Commons Attribution License, which permits unrestricted use, distribution, and reproduction in any medium, provided the original work is properly cited.

In this paper general solution to the problem of finding maximally flat waveforms with finite number of harmonics (maximally flat trigonometric polynomials) is provided. Waveform coefficients are expressed in closed form as functions of harmonic orders. Two special cases of maximally flat waveforms (so-called maximally flat even harmonic and maximally flat odd harmonic waveforms), which proved to play an important role in class- $F$ and inverse class- $F$ power amplifier (PA) operations, are also considered. For these two special types of waveforms, coefficients are expressed as functions of two parameters only. Closed form expressions for efficiency and power output capability of class- $F$ and inverse class- $F$ PA operations with maximally flat waveforms are also provided as explicit functions of number of a harmonics.

\section{Introduction}

Roughly speaking, maximally flat waveform (maximally flat trigonometric polynomial) of a family of waveforms is a waveform which possesses degenerate critical point with highest degree (maximally degenerate critical point), among all members of the family. At the maximally degenerate critical point, maximum possible consecutive derivatives of waveform are equal to zero, starting from the first. The existence of maximally flat trigonometric polynomials, according to our best knowledge, has been reported for the first time in [1] (in Serbian), in the context of catastrophes in parameter space of trigonometric polynomials with two harmonics of arbitrary order.

In the context of the analysis of power amplifiers (PAs) in electrical engineering, the benefits of flattening of the bottom of voltage waveform were known as early as 1919 (e.g., see [2]), but the credit for the first comprehensive usage of maximally flat waveforms, in this context, goes to Raab [2]. In this pioneering paper a subclass of maximally flat waveforms with fundamental harmonic and two prescribed higher harmonics $P, Q \leq 5$, that can be obtained from cosine polynomials after shifting by $\pi / 2$, has been considered. Grebennikov and Sokal [3] extended Raab's result up to seventh harmonic. In [4], the coefficients of maximally flat trigonometric polynomials with fundamental and additional two harmonics with arbitrary order $P$ and $Q$ have been provided in closed form as functions of $P$ and $Q$. A closed form solution to the general case of maximally flat cosine polynomials with $N$ consecutive harmonics has been presented in [5].

In this paper, we consider the general case of maximally flat trigonometric polynomials. In Section 2 we provide general solution to the problem of finding closed form representations of maximally flat trigonometric polynomials using rigorous mathematical tools. Two special subclasses of maximally flat trigonometric polynomials with natural application in class- $F$ and inverse class- $F$ PA operations are considered in Section 3. In Section 4, based on results of Section 3, closed form expressions of efficiency and poweroutput capability of class- $F$ and inverse class- $F$ PA with maximally flat waveforms are derived.

Motivating Example. Let us consider the following family with two parameters, consisting of waveforms with first and third harmonics:

$$
f(\tau ; A, \alpha)=\cos \tau+A \cos (3 \tau+\alpha),
$$




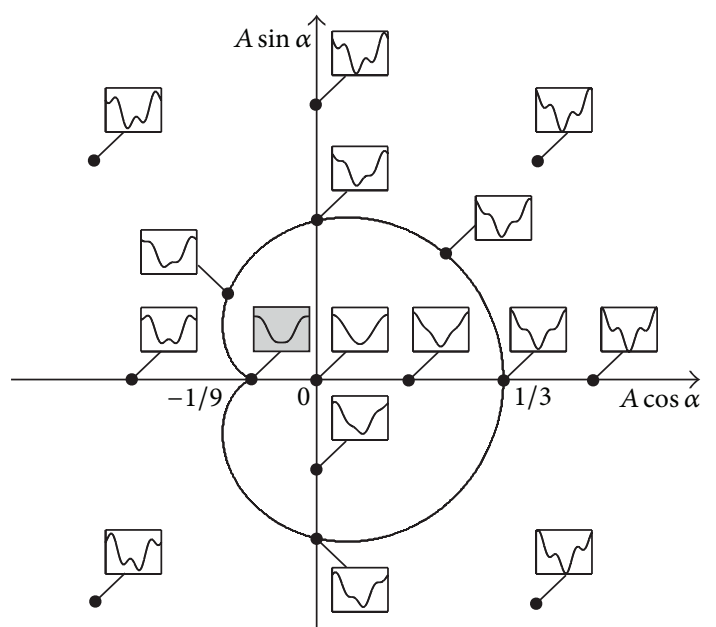

Figure 1: Parameter space $(A \cos \alpha, A \sin \alpha)$ and waveforms of the family $f(\tau ; A, \alpha)=\cos \tau+A \cos (3 \tau+\alpha)$.

and corresponding set of 3-tiples $\left(\tau_{d}, A, \alpha\right)$ for which both first and second derivatives of the waveform $f(\tau ; A, \alpha)$ are equal to zero:

$$
f^{(1)}\left(\tau_{d} ; A, \alpha\right)=0, \quad f^{(2)}\left(\tau_{d} ; A, \alpha\right)=0 .
$$

In this case, we say that $\tau_{d}$ is a degenerate critical point of the waveform $f(\tau ; A, \alpha)$. The pairs $(A, \alpha)$ that correspond to the waveforms with degenerate critical points form socalled catastrophe set, illustrated in Figure 1 by solid line. For the family (1), the corresponding catastrophe set divides the parameter space $(A \cos \alpha, A \sin \alpha)$ into three disjoint subsets (inner and outer part of the solid line and solid line itself) and helps in making classification of the zoology of waveforms $f(\tau ; A, \alpha)$. Waveforms that correspond to the inner points have one minimum and one maximum, whereas the waveforms that correspond to the outer points have three minima and three maxima. Points on the solid line correspond to the waveforms with one minimum, one maximum and two inflection points. The cusp point $(A=1 / 9, \alpha=\pi)$ corresponds to the maximally flat waveform shown in the shadowed frame in Figure 1. The fact that maximally flat waveforms are related to the cusp points of the catastrophe set illustrates that they are rather exceptional.

\section{General Case of Maximally Flat Waveforms}

Critical point of a waveform is a point at which first derivative is equal to zero. Critical point of waveform is degenerate if at least first two derivatives are equal to zero. An integer $r \geq 2$ is said to be the degree of degeneracy of critical point if first $r$ consecutive derivatives are equal to zero and $(r+1)$ th derivative is not.

In this Section we consider the following problem: find a waveform from the family

$$
f_{N}(\tau)=F_{0}+\sum_{i=1}^{N} F_{i} \cos \left(n_{i} \tau+\varphi_{i}\right), \quad N \geq 1
$$

which possess critical point of highest degree of degeneracy. Such a waveform is said to be maximally flat waveform of the family. We can assume without loss of generality that $F_{i}>0$ and $n_{j}>n_{i}$. Notice that set of positive integers $n_{i}$ defines the family.

It is obvious that the existence of the maximally degenerate critical point is invariant of the value of $F_{0}$ and therefore, without loss of generality we can assume that the waveform is equal to zero at the maximally degenerate critical point. It is also clear that existence of the maximally degenerate critical point is invariant of the translation along the $\tau$ axis and consequently maximally degenerate critical point can be placed at any point along $\tau$-axis. In what follows it is convenient to assume that maximally degenerate critical point is $\tau_{0}=0$.

The above problem of finding waveform $f_{N}(\tau)$ of the family (3) with maximally degenerate critical point, providing that the value of the waveform equals zero at this degenerate critical point, can be replaced by an equivalent problem of finding a nonzero waveform $f_{N}(\tau)$ of the family (3) such that

$$
\begin{gathered}
f_{N}(0)=0, \\
f_{N}^{(r)}(0)=0, \quad r=1, \ldots, r_{\max },
\end{gathered}
$$

where $f_{N}^{(r)}$ denotes $r$ th derivative of the waveform $f_{N}$, and $r_{\max }$ is maximum number of relations (5) which do not contradict (4).

Lemma 1. For nonzero waveform $f_{N}(\tau)$ of the family (3) which satisfies (4) and (5), the following relations hold:

$$
\begin{gathered}
F_{0} \neq 0, \\
r_{\text {max }}=2 N-1 .
\end{gathered}
$$

Remark 2. From (7) it is obvious that $r_{\max }$ is odd number which further implies that at maximally degenerate critical point $f_{N}(\tau)$ has either minimum or maximum.

Proof. In terms of new variables

$$
\begin{aligned}
& a_{i}=F_{i} \cos \varphi_{i}, \quad i=1, \ldots, N, \\
& b_{i}=F_{i} \sin \varphi_{i}, \quad i=1, \ldots, N,
\end{aligned}
$$

system (4)-(5) for $r_{\max }=2 N-1$ decomposes in two independent subsystems:

$$
\begin{gathered}
\sum_{i=1}^{N} a_{i}=-F_{0}, \\
\sum_{i=1}^{N} n_{i}^{2 q} a_{i}=0, \quad q=1, \ldots, N-1,
\end{gathered}
$$

(written in terms of variables $a_{i}$ only) and

$$
\sum_{i=1}^{N} n_{i}^{2 q-1} b_{i}=0, \quad q=1, \ldots, N
$$

(written in terms of variables $b_{i}$ only). 
The matrices of subsystems (10)-(11) and (12) are

$$
\begin{gathered}
M_{a}=\left[\begin{array}{ccccccc}
1 & \cdots & 1 & 1 & 1 & \cdots & 1 \\
n_{1}^{2} & \cdots & n_{i-1}^{2} & n_{i}^{2} & n_{i+1}^{2} & \cdots & n_{N}^{2} \\
\vdots & \ddots & \vdots & \vdots & \vdots & \ddots & \vdots \\
n_{1}^{2 N-2} & \cdots & n_{i-1}^{2 N-2} & n_{i}^{2 N-2} & n_{i+1}^{2 N-2} & \cdots & n_{N}^{2 N-2}
\end{array}\right], \\
M_{b}=\left[\begin{array}{ccccccc}
n_{1} & \cdots & n_{i-1} & n_{i} & n_{i+1} & \cdots & n_{N} \\
n_{1}^{3} & \cdots & n_{i-1}^{3} & n_{i}^{3} & n_{i+1}^{3} & \cdots & n_{N}^{3} \\
\vdots & \ddots & \vdots & \vdots & \vdots & \ddots & \vdots \\
n_{1}^{2 N-1} & \cdots & n_{i-1}^{2 N-1} & n_{i}^{2 N-1} & n_{i+1}^{2 N-1} & \cdots & n_{N}^{2 N-1}
\end{array}\right],
\end{gathered}
$$

respectively. Matrices $M_{a}$ and $M_{b}$ are both Vandermonde matrices and their determinants are

$$
\begin{aligned}
& \operatorname{det}\left(M_{a}\right)=\prod_{1 \leq i<j \leq N}\left(n_{j}^{2}-n_{i}^{2}\right), \\
& \operatorname{det}\left(M_{b}\right)=\operatorname{det}\left(M_{a}\right) \prod_{1 \leq j \leq N} n_{j},
\end{aligned}
$$

which are clearly nonzero. Therefore, each subsystem (10)(11)and (12) has unique solution which implies that $r_{\max } \geq$ $2 N-1$.

It is obvious that homogeneous subsystem (12) in terms of $b_{i}$ has trivial solution as follows:

$$
b_{i}=0, \quad i=1, \ldots, N,
$$

and it is unique.

Let us first show that (6) holds. Suppose in the contrary that $F_{0}=0$. Then subsystem (10)-(11) has also trivial solution only, which implies that waveform (3) is identically equal to zero.

Let us now show that (7) also holds. We already have proved that $r_{\max } \geq 2 N-1$ and we are going to show that assumption $r_{\max }=2 \mathrm{~N}$ leads to contradiction. For $r_{\max }=2 \mathrm{~N}$, the corresponding subsystem can be obtained from (10)-(11) by including additional equation $f_{N}^{(2 N)}(0)=0$, which in terms of variables (8), can be written in the form

$$
\sum_{i=1}^{N} n_{i}^{2 N} a_{i}=0 .
$$

Determinant of subsystem consisting of equations (11) and (17) equals $\operatorname{det}\left(M_{a}\right) \prod_{1 \leq j \leq N} n_{j}^{2}$ and it is clearly nonzero. Hence, this subsystem has only trivial solution, which contradicts (10) since $F_{0} \neq 0$. This completes the proof.

As a consequence of Lemma 1, the problem of finding nonzero waveform $f_{N}(\tau)$ of the family (3) satisfying (4) and (5) can be reformulated as follows: find a nonzero waveform from the family (3) such that

$$
\begin{gathered}
f_{N}(0)=0 \\
f_{N}^{(r)}(0)=0, \quad r=1, \ldots, 2 N-1 .
\end{gathered}
$$

In terms of variables $a_{i}$ and $b_{i}$ (see (8) and (9)) system (18) can be transformed into the system (10)-(12). In what follows we will provide closed form solution of this system, which leads to the maximally flat waveform.

Proposition 3. Maximally flat waveform of the family (3) with maximally degenerate critical point at $\tau_{0}=0$, having zero value at this point is fully described by the following set of parameters:

$$
\begin{gathered}
F_{i}=\left|F_{0}\right|\left[\prod_{1 \leq j \leq i-1} \frac{n_{j}^{2}}{\left(n_{i}^{2}-n_{j}^{2}\right)}\right] \cdot\left[\prod_{i+1 \leq j \leq N} \frac{n_{j}^{2}}{\left(n_{j}^{2}-n_{i}^{2}\right)}\right], \\
i=1, \ldots, N, \\
\varphi_{i}= \begin{cases}0 & i \text { is even }, \quad i=1, \ldots, N, \text { if } F_{0}>0, \\
\pi & i \text { is odd },\end{cases} \\
\varphi_{i}= \begin{cases}\pi & i \text { is even }, \quad i=1, \ldots, N, \text { if } F_{0}<0 . \\
0 & i \text { is odd },\end{cases}
\end{gathered}
$$

Proof. Let us find the solution of the system (10)-(12). Using Cramer's rule, from subsystem (10)-(11) it follows that

$$
\begin{aligned}
a_{i}= & -F_{0} \frac{(-1)^{1+i} M_{1 i}}{\operatorname{det}\left(M_{a}\right)}=(-1)^{i} F_{0}\left[\prod_{1 \leq j \leq i-1} \frac{n_{j}^{2}}{\left(n_{i}^{2}-n_{j}^{2}\right)}\right] \\
& \cdot\left[\prod_{i+1 \leq j \leq N} \frac{n_{j}^{2}}{\left(n_{j}^{2}-n_{i}^{2}\right)}\right], \quad i=1, \ldots, N,
\end{aligned}
$$

where $M_{1 i}$ is (1i)th minor of the matrix (13). Notice that the first product is empty for $i=1$ and second for $i=N$ (by definition an empty product is equal to 1). From (8), (9), (16), (20) and $F_{i}>0$ immediately follows (19), which completes the proof.

Example 4. The following two waveforms presented in Figure 2:

$$
\begin{gathered}
f_{(1,3,4)}(\tau)=1-\frac{6}{5} \cos \tau+\frac{2}{7} \cos 3 \tau-\frac{3}{35} \cos 4 \tau, \\
f_{(2,4,5)}(\tau)=1-\frac{100}{63} \cos 2 \tau+\frac{25}{27} \cos 4 \tau-\frac{64}{189} \cos 5 \tau,
\end{gathered}
$$

are maximally flat waveforms of the family with 1st, 3rd and 4 th harmonic and family with 2nd, 4th and 5th harmonic, respectively, with maximally degenerate critical point at $\tau_{0}=$ 0.

\section{Two Special Cases: Maximally Flat Even and Odd Harmonic Waveforms}

The following are two special types of waveforms:

$$
\begin{gathered}
w_{\text {even }}(\tau)=1-A_{1} \cos \tau-\sum_{m=1}^{M} A_{2 m} \cos (2 m \tau), \\
w_{\text {odd }}(\tau)=1-B_{1} \cos \tau-\sum_{k=1}^{K} B_{(2 k+1)} \cos (2 k+1) \tau,
\end{gathered}
$$




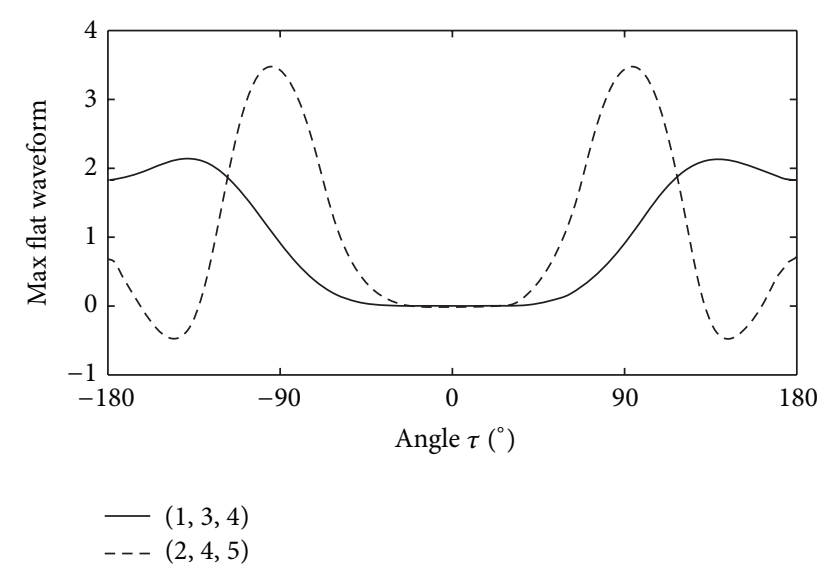

FIGURE 2: Examples of two maximally flat waveforms.

where $A_{1}>0$ and $B_{1}>0$, which are of particular interest in PA efficiency analysis. Waveform (22) which contains dc component, fundamental harmonic, and $M$ consecutive even harmonics is said to be an even harmonic waveform. Waveform (23) which contains dc component, fundamental, and $K$ consecutive odd harmonics is said to be an odd harmonic waveform.

In what follows we assume that $w_{\text {even }}(\tau)$ and $w_{\text {odd }}(\tau)$ refer to maximally flat even and odd harmonic waveforms, respectively, with maximally degenerate critical point at $\tau_{0}=$ 0 and zero value at this point.

In this Section, we show that maximally flat even and odd harmonic waveforms of the form (22) and (23) have global minimum at maximally degenerate critical point. Such a minimum we will call "maximally flat minimum." Since the waveforms have zero values at this point, it immediately follows that they are nonnegative. We also derive basic parameters of these waveforms.

The coefficients of maximally flat even harmonic waveform (22), with maximally degenerate critical point at $\tau_{0}=0$ and zero value at this point, can be obtained straightforward from (20) for $N=M+1, n_{1}=1, F_{0}=1, A_{1}=-a_{1}, n_{m+1}=$ $2 m$, and $A_{2 m}=-a_{m+1}$ for $m=1, \ldots, M$ :

$$
\begin{gathered}
A_{1}=\frac{((2 M) ! !)^{2}}{(2 M-1) ! !(2 M+1) ! !}, \\
A_{2 m}=\frac{(-1)^{m}}{\left(4 m^{2}-1\right)} \frac{2(M !)^{2}}{(M-m) !(M+m) !}, \\
m=1, \ldots, M .
\end{gathered}
$$

The coefficients of maximally flat odd harmonic waveform (23), with maximally degenerate critical point at $\tau_{0}=0$ and zero value at this point, can be obtained straightforward from (20) for $N=K+1, n_{k+1}=2 k+1, F_{0}=1$, and $B_{(2 k+1)}=-a_{k+1}$ for $k=0, \ldots, K$ :

$$
\begin{array}{r}
B_{(2 k+1)}=\frac{(-1)^{k}}{(2 k+1)} \cdot \frac{((2 K+1) ! !)^{2}}{4^{K}(K-k) !(K+k+1) !}, \\
k=0, \ldots, K .
\end{array}
$$

Let us introduce "duals" of the waveforms (22) and (23):

$$
\begin{gathered}
w_{\text {even }}^{*}(\tau)=1+A_{1}^{*} \cos \tau+\sum_{m=1}^{M} A_{2 m}^{*} \cos (2 m \tau), \\
w_{\text {odd }}^{*}(\tau)=1+B_{1}^{*} \cos \tau+\sum_{k=1}^{K} B_{(2 k+1)}^{*} \cos (2 k+1) \tau,
\end{gathered}
$$

which is defined as $w_{\text {even }}^{*}(\tau)=w_{\text {even }}(\tau+\pi)$ and $w_{\text {odd }}^{*}(\tau)=$ $w_{\text {odd }}(\tau+\pi)$, respectively. It is easy to see that the following relations between the coefficients of waveforms (22)-(23) and their duals hold:

$$
\begin{gathered}
A_{1}^{*}=A_{1}, \quad A_{2 m}^{*}=-A_{2 m}, \\
B_{1}^{*}=B_{1}, \quad B_{(2 k+1)}^{*}=B_{(2 k+1)} .
\end{gathered}
$$

Notice that $w_{\text {even }}(\tau)$ and $w_{\text {odd }}(\tau)$ have maximally degenerate critical point at $\tau_{0}=0$, which implies that their duals $w_{\text {even }}^{*}(\tau)$ and $w_{\text {odd }}^{*}(\tau)$ have maximally degenerate critical point at $\tau_{0}=$ $\pi$.

3.1. Maximally Flat Even Harmonic Waveform. In this subsection we show that maximally flat even harmonic waveform is nonnegative and has global minimum at maximally degenerate critical point (Proposition 6). We also provide closed form expression for basic waveform parameters $\gamma_{\text {even }}$ and $\delta_{\text {even }}$ in terms of number of even harmonics. The parameter $\gamma_{\text {even }}$ is defined as the amplitude of fundamental harmonic relative to dc component of the even harmonic waveform, whereas $\delta_{\text {even }}$ is defined as maximum value of the waveform relative to dc component (e.g., see [2]):

$$
\gamma_{\text {even }}=A_{1}, \quad \delta_{\text {even }}=\max _{\tau} w_{\text {even }}(\tau) .
$$

According to (24), parameter $\gamma_{\text {even }}$ is

$$
\begin{aligned}
\gamma_{\text {even }} & =A_{1}=\frac{((2 M) ! !)^{2}}{(2 M-1) ! !(2 M+1) ! !} \\
& =\frac{16^{M}}{2 M+1}\left(\frac{(M !)^{2}}{(2 M) !}\right)^{2} .
\end{aligned}
$$

Notice that by definition $0 ! !=1$ and $(-1) ! !=1$. Therefore, (30) yields $\gamma_{\text {even }}=1$ for $M=0$. The relation (30) shows that $\gamma_{\text {even }}$ increases by increasing $M$ (see Figure 3 ). When $M$ tends to infinity, parameter $\gamma_{\text {even }}$ equals to Wallis product; that is,

$$
\lim _{M \rightarrow \infty} \gamma_{\text {even }}=\prod_{n=1}^{\infty} \frac{4 n^{2}}{(2 n-1)(2 n+1)}=\frac{\pi}{2} .
$$

We first prove the following statement:

Lemma 5. Maximally flat even harmonic waveform with maximally degenerate critical point at $\tau_{0}=0$ and zero value at this point can be expressed in the following form:

$$
\begin{array}{r}
w_{\text {even }}(\tau)=\gamma_{\text {even }}\left(1-\cos \tau-\sum_{m=1}^{M} \frac{(2 m-3) ! !}{(2 m) ! !}(\sin \tau)^{2 m}\right), \\
M \geq 0,
\end{array}
$$

where $\gamma_{\text {even }}$ is given by (30). 


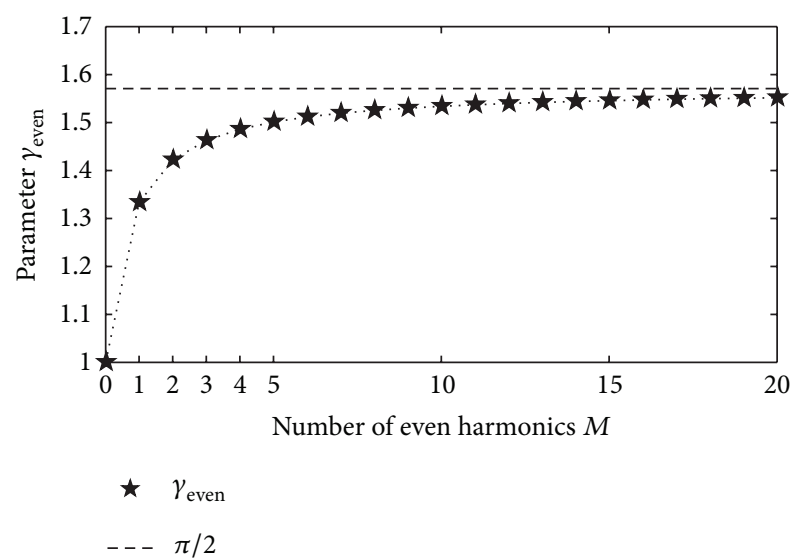

FIGURE 3: Waveform parameter $\gamma_{\text {even }}$ for maximally flat even harmonic waveform as function of number of even harmonics $M$.

Proof. Taking into account (30) and (25), maximally flat even harmonic waveform (22) with maximally degenerate critical point at $\tau_{0}=0$ can be expressed as

$$
w_{\text {even }}(\tau)=\gamma_{\text {even }}\left(-\cos \tau+S_{M}(\tau)\right)
$$

where $S_{0}(\tau)=1$ and

$$
\begin{aligned}
S_{M}(\tau)= & \frac{(2 M+1) ! !}{4^{M}(2 M) ! !} \\
& \times\left[\left(\begin{array}{c}
2 M \\
M
\end{array}\right)-2 \sum_{m=1}^{M}\left(\begin{array}{c}
2 M \\
M-m
\end{array}\right) \frac{(-1)^{m} \cos (2 m \tau)}{\left(4 m^{2}-1\right)}\right],
\end{aligned}
$$

The expression $4^{M}(\sin \tau)^{2 M}=(-1)^{M}\left(e^{-j \tau}-e^{j \tau}\right)^{2 M}$, where $j=\sqrt{-1}$, in an expanded form reads

$$
4^{M}(\sin \tau)^{2 M}=\sum_{n=0}^{2 M}\left(\begin{array}{c}
2 M \\
n
\end{array}\right)(-1)^{M-n} e^{j 2(M-n) \tau}
$$

Substitution of $n=M-m$ into (35) yields

$$
4^{M}(\sin \tau)^{2 M}=\sum_{m=-M}^{M}\left(\begin{array}{c}
2 M \\
M-m
\end{array}\right)(-1)^{m} e^{j 2 m \tau}
$$

that is,

$$
\begin{aligned}
4^{M}(\sin \tau)^{2 M}= & \left(\begin{array}{c}
2 M \\
M
\end{array}\right) \\
& +2 \sum_{m=1}^{M}\left(\begin{array}{c}
2 M \\
M-m
\end{array}\right)(-1)^{m} \cos (2 m \tau) .
\end{aligned}
$$

Since

$$
\begin{aligned}
S_{M}(\tau) & -S_{M-1}(\tau) \\
= & -\frac{(2 M-3) ! !}{4^{M}(2 M) ! !} \\
& \times\left[\left(\begin{array}{c}
2 M \\
M
\end{array}\right)+2 \sum_{m=1}^{M}\left(\begin{array}{c}
2 M \\
M-m
\end{array}\right)(-1)^{m} \cos (2 m \tau)\right],
\end{aligned}
$$

using (37), we obtain

$$
S_{M}(\tau)-S_{M-1}(\tau)=-\frac{(2 M-3) ! !}{(2 M) ! !}(\sin \tau)^{2 M}, \quad S_{0}(\tau)=1 .
$$

The solution of recursive relation (39) reads

$$
S_{M}(\tau)=1-\sum_{m=1}^{M} \frac{(2 m-3) ! !}{(2 m) ! !}(\sin \tau)^{2 m}, \quad M \geq 1 .
$$

Substituting (40) into (33), we finally obtain (32), which completes the proof.

Using Lemma 5, we prove the following proposition.

Proposition 6. Maximally flat even harmonic waveform with maximally degenerate critical point at $\tau_{0}=0$ and zero value at this point is nonnegative and has unique global minimum at $\tau_{0}=0$.

Proof. The binomial series

$$
\left(1-x^{2}\right)^{1 / 2}=1-\sum_{m=1}^{\infty} \frac{(2 m-3) ! !}{(2 m) ! !} x^{2 m}, \quad-1 \leq x \leq 1,
$$

for $x=\sin \tau$ reads

$$
|\cos \tau|=\left(1-\sin ^{2} \tau\right)^{1 / 2}=1-\sum_{m=1}^{\infty} \frac{(2 m-3) ! !}{(2 m) ! !}(\sin \tau)^{2 m} .
$$

By comparison of (32) with (42), we conclude that

$$
w_{\text {even }}(\tau) \geq \gamma_{\text {even }}(-\cos \tau+|\cos \tau|) \geq 0,
$$

and therefore the maximally flat even harmonic waveform is nonnegative.

From (43) and (32), it is obvious that $w_{\text {even }}(\tau)$ is equal to zero if and only if $\tau=0$; that is, it has unique global minimum at maximally degenerate critical point $\tau_{0}=0$. This completes the proof.

Remark 7. Since $\gamma_{\text {even }}>0$, it is obvious that the maximum value of (32) is achieved for $\sin \tau=0$ and $\cos \tau=-1$, that is, for $\tau=\pi$, only. Thus,

$$
\delta_{\text {even }}=\max _{\tau} w_{\text {even }}(\tau)=w_{\text {even }}(\pi)=2 \gamma_{\text {even }} .
$$

Raab [2] also pointed out the relation (44). 


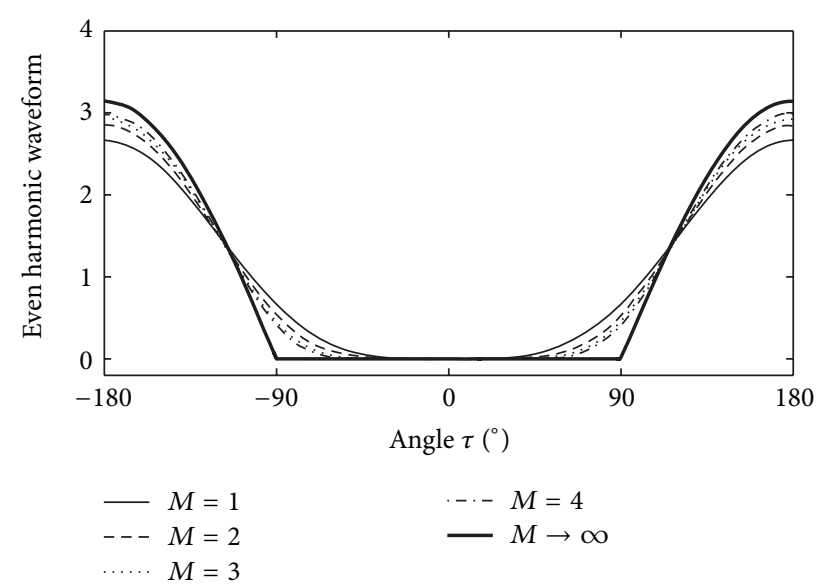

FIGURE 4: Maximally flat even harmonic waveforms.

Remark 8. When $M$ tends to infinity, according to (32), (42), and (31) it follows that

$$
\lim _{M \rightarrow \infty} w_{\text {even }}(\tau)=\frac{\pi}{2}(-\cos \tau+|\cos \tau|)
$$

Clearly, (45) is a "half-sine" waveform.

Maximally flat even harmonic waveforms, including limit waveform $(M \rightarrow \infty)$, are presented in Figure 4 .

3.2. Maximally Flat Odd Harmonic Waveform. In this subsection, we show that maximally flat odd harmonic waveform is nonnegative and has global minimum at maximally degenerate critical point (Proposition 10). We also provide closed form expression for basic waveform parameters $\gamma_{\text {odd }}$ and $\delta_{\text {odd }}$ in terms of number of odd harmonics. The parameter $\gamma_{\text {odd }}$ is defined as the amplitude of fundamental harmonic relative to dc component of the odd harmonic waveform, whereas $\delta_{\text {odd }}$ is defined as maximum value of the waveform relative to $\mathrm{dc}$ component (e.g., see [2]):

$$
\gamma_{\text {odd }}=B_{1}, \quad \delta_{\text {odd }}=\max _{\tau} w_{\text {odd }}(\tau)
$$

From (26) for $k=0$, we obtain

$$
\gamma_{\text {odd }}=B_{1}=2 \frac{((2 K+1) ! !)^{2}}{(2 K) ! !(2 K+2) ! !}=\frac{K+1}{16^{K}}\left(\begin{array}{c}
2 K+1 \\
K
\end{array}\right)^{2}
$$

Notice that (47) yields $\gamma_{\text {odd }}=1$ for $K=0$. The relation (47) shows that $\gamma_{\text {odd }}$ increases by increasing $K$ (see Figure 5 ). When $K$ tends to infinity, from (47), according to (31), $\gamma_{\text {odd }}$ tends to

$$
\lim _{K \rightarrow \infty} \gamma_{\text {odd }}=\lim _{K \rightarrow \infty}\left(\frac{2 K+1}{K+1} \prod_{n=1}^{K} \frac{(2 n-1)(2 n+1)}{4 n^{2}}\right)=\frac{4}{\pi} .
$$

Let us first prove the following Lemma.

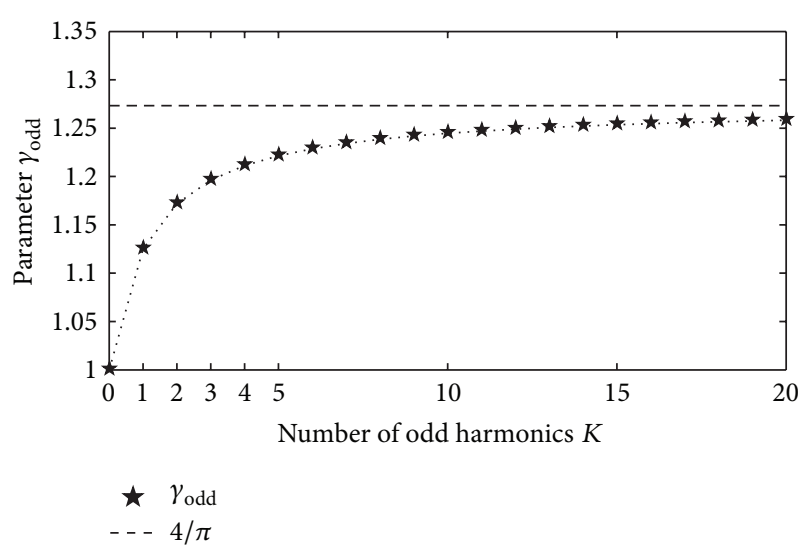

FIGURE 5: Waveform parameter $\gamma_{\text {odd }}$ for maximally flat odd harmonic waveform as function of number of odd harmonics $K$.

Lemma 9. Maximally flat odd harmonic waveform with maximally degenerate critical point at $\tau_{0}=0$ and zero value at this point can be expressed as

$$
\begin{array}{r}
w_{\text {odd }}(\tau)=1-\cos \tau\left(1+\sum_{k=1}^{K} \frac{(2 k-1) ! !}{(2 k) ! !}(\sin \tau)^{2 k}\right) \\
K \geq 0 .
\end{array}
$$

Proof. Taking into account (26), maximally flat odd harmonic waveform (23) with maximally degenerate critical point at $\tau_{0}=0$ can be expressed as

$$
w_{\text {odd }}(\tau)=1-S_{K}(\tau)
$$

where $S_{0}(\tau)=\cos \tau$ and

$$
S_{K}(\tau)=\frac{(2 K+1) ! !}{4^{K}(2 K) ! !} \sum_{k=0}^{K}\left(\begin{array}{c}
2 K+1 \\
K-k
\end{array}\right) \frac{(-1)^{k}}{(2 k+1)} \cos (2 k+1) \tau .
$$

Hence, difference $S_{K}(\tau)-S_{K-1}(\tau)$ is equal to

$$
\begin{aligned}
S_{K}(\tau) & -S_{K-1}(\tau) \\
= & \frac{1}{(2 K+1)} \frac{(2 K-1) ! !}{4^{K}(2 K) ! !} \\
& \times \sum_{k=0}^{K}\left(\begin{array}{c}
2 K+1 \\
K-k
\end{array}\right)(-1)^{k}(2 k+1) \cos (2 k+1) \tau .
\end{aligned}
$$

The expression

$$
4^{K}(\sin \tau)^{2 K+1}=\frac{1}{2} j(-1)^{K} e^{j(2 K+1) \tau}\left(e^{-j 2 \tau}-1\right)^{2 K+1},
$$

in an expanded form reads

$$
4^{K}(\sin \tau)^{2 K+1}=-\frac{1}{2} j \sum_{n=0}^{2 K+1}\left(\begin{array}{c}
2 K+1 \\
n
\end{array}\right)(-1)^{K-n} e^{j(2 K+1-2 n) \tau} .
$$


Substitution of $k=K-n$ into (54) yields

$$
4^{K}(\sin \tau)^{2 K+1}=-\frac{1}{2} j \sum_{k=-(K+1)}^{K}\left(\begin{array}{c}
2 K+1 \\
K-k
\end{array}\right)(-1)^{k} e^{j(2 k+1) \tau},
$$

that is,

$$
4^{K}(\sin \tau)^{2 K+1}=\sum_{k=0}^{K}\left(\begin{array}{c}
2 K+1 \\
K-k
\end{array}\right)(-1)^{k} \sin (2 k+1) \tau .
$$

By differentiating (56) and comparing the resulting expression with (52), we obtain

$$
\begin{gathered}
S_{K}(\tau)=S_{K-1}(\tau)+\frac{(2 K-1) ! !}{(2 K) ! !}(\sin \tau)^{2 K} \cos \tau, \\
S_{0}(\tau)=\cos \tau .
\end{gathered}
$$

Solving recurrent relation (57), we finally obtain (49), which completes the proof.

Using Lemma 9, we prove the following proposition.

Proposition 10. Maximally flat odd harmonic waveform with maximally degenerate critical point at $\tau_{0}=0$ and zero value at this point is nonnegative and has unique global minimum at $\tau_{0}=0$.

Proof. First derivative of (49) reads

$$
w_{\text {odd }}^{(1)}(\tau)=\frac{(2 K+1) ! !}{(2 K) ! !}(\sin \tau)^{2 K+1} .
$$

It is obvious that (58) has only two zeros; namely, $\tau=0$ and $\tau=\pi$. Therefore, (49) has only one minimum and only one maximum. Since $w_{\text {odd }}(0)=0$ and $w_{\text {odd }}(\pi)=2$ it follows that $w_{\text {odd }}(\tau)$ is nonnegative and has unique global minimum at maximally degenerate critical point $\tau_{0}=0$. This completes the proof.

Remark 11. As it was pointed out earlier, the maximum value of (49) is achieved for $\tau=\pi$, which implies

$$
\delta_{\text {odd }}=\max _{\tau} w_{\text {odd }}(\tau)=w_{\text {odd }}(\pi)=2 .
$$

Raab [2] also pointed out the existence of relation (59).

Remark 12. Although the binomial series

$$
\begin{array}{r}
\left(1-x^{2}\right)^{-1 / 2}=1+\sum_{k=1}^{\infty} \frac{(2 k-1) ! !}{(2 k) ! !} x^{2 k} \\
-1<x<1,
\end{array}
$$

does not converge for $x^{2}=1$, the above formula holds when $x^{2}$ tends to 1 from the left side (Abel's limit theorem). Since $\cos \tau=\operatorname{sgn}(\cos \tau) \cdot|\cos \tau|$, according to the definition of the sign function, from (49), (60), and (48), for $x=\sin \tau$, it follows that

$$
\lim _{K \rightarrow \infty} w_{\text {odd }}(\tau)=1-\operatorname{sgn}(\cos \tau)
$$

Clearly, (61) is a square waveform.

Maximally flat odd harmonic waveforms, including limit waveform $(K \rightarrow \infty)$, are presented in Figure 6 .

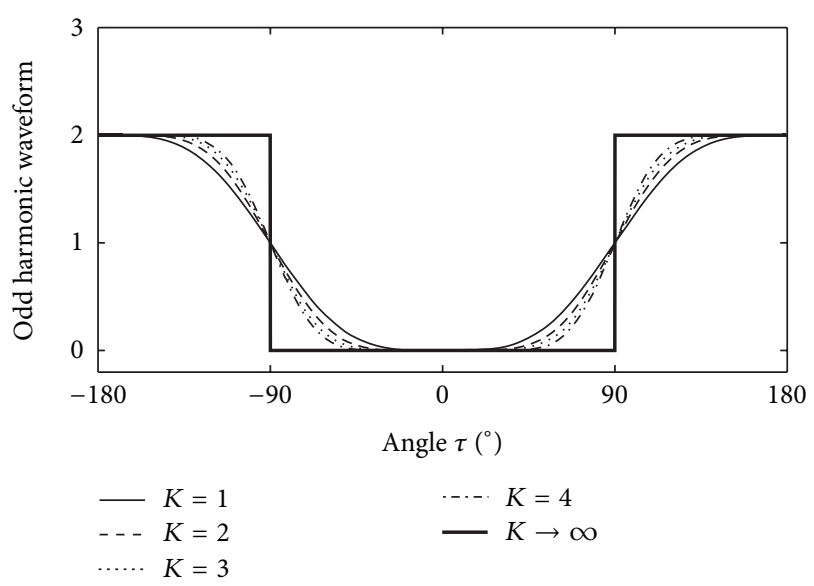

FIGURE 6: Maximally flat odd harmonic waveforms.

\section{Efficiency and Power-Output Capability of Class- $F$ and Inverse Class- $F$ PA with Maximally Flat Waveforms}

There is a continuous interest in shaping current and voltage waveforms (e.g., see [2-7]). The case when both voltage and current waveforms contain only finite number of harmonics is of particular importance (e.g., see [2, 3, 5-7]). It has been notified $[2,3]$ that maximally flat waveforms could offer an approximate solution for current and voltage waveforms of finite harmonic class- $F$ and inverse class- $F$ PA.

In this Section, even harmonic and odd harmonic waveforms with maximally flat global minimum combined with their duals play central role in finding efficiency and poweroutput capability of class-F PA and inverse class-PA with maximally flat waveforms.

Raab [2] noticed that maximally flat waveforms could offer a good approximation for current and voltage waveforms for class- $F$ PA operation. He investigated maximally flat waveforms up to fifth harmonic, and efficiencies of class$F$ PA operation for various combinations of harmonics up to five.

In this Section, we provide closed form expression for the efficiency of class- $F$ PA and inverse class- $F$ PA with maximally flat waveforms. We also provide proof of an interesting statement with practical implications saying that there is more benefit in consecutive inclusion of harmonics alternatively in current and voltage waveforms, than in inclusion of several harmonics in one waveform only. This statement was originally formulated in [2] based on the consideration of instances up to fifth harmonic, without general proof. When all even harmonics up to $N$ are included in current (voltage) waveform and all odd harmonics up to $N$ are included in voltage (current) waveform, we say that we are dealing with class- $F$ (inverse class- $F$ ) PA with $N$ harmonics. For this case, we show that the efficiency of the class-F PA and the inverse class- $F$ PA with maximally flat waveforms is $\eta_{N \text {,flat }}=N /(N+1)$. We would like to call attention to the fact that this efficiency is identical to the efficiency of finite harmonic class- $C$ PA with maximally flat current waveform, when it contains first $N$ harmonics [5]. 


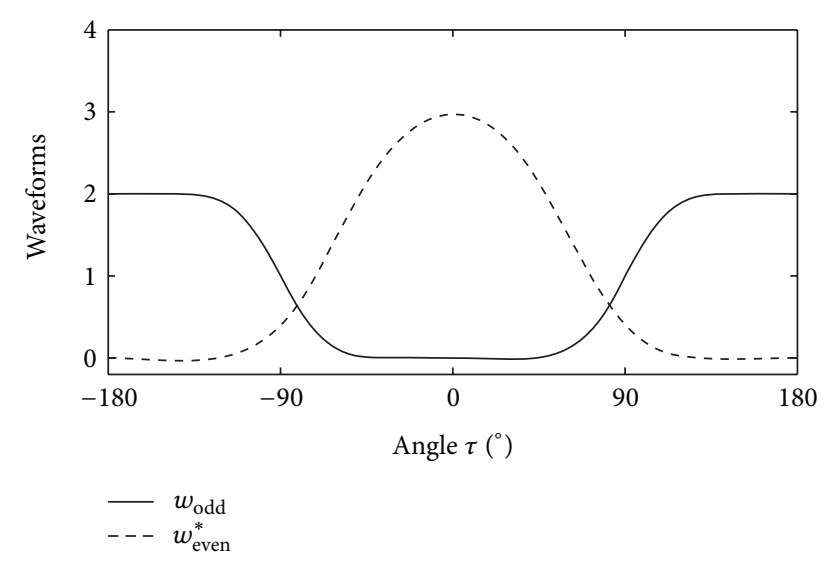

Figure 7: Pair of maximally flat waveforms $\left(w_{\text {even }}^{*}, w_{\text {odd }}\right)$ for class- $F$ PA and inverse class- $F$ PA for $N=9$.

For class- $F$ PA and inverse class- $F$ PA one of the waveforms possesses minimum at $\tau_{0}$ and the other at $\tau_{0}+\pi$. Without loss of generality, we assume $\tau_{0}=0$.

For the purpose of the analysis of efficiency for class- $F$ PA and inverse class- $F$ PA with maximally flat waveforms we introduce either pair $\left(w_{\text {even }}, w_{\text {odd }}^{*}\right)$ or $\left(w_{\text {even }}^{*}, w_{\text {odd }}\right)$ of waveforms, where $w_{\text {even }}, w_{\text {odd }}$, and their duals $w_{\text {even }}^{*}, w_{\text {odd }}^{*}$ are defined in Section 3.

For class- $F$ PA with maximally flat waveforms, the pair of current and voltage waveforms can be expressed in two ways:

$$
i(\tau)=I_{\mathrm{dc}} w_{\text {even }}(\tau), \quad v(\tau)=V_{\mathrm{dc}} w_{\text {odd }}^{*}(\tau),
$$

or

$$
i(\tau)=I_{\mathrm{dc}} w_{\text {even }}^{*}(\tau), \quad v(\tau)=V_{\mathrm{dc}} w_{\mathrm{odd}}(\tau)
$$

where $\tau$ stands for $\omega t, I_{\mathrm{dc}}>0$ and $V_{\mathrm{dc}}>0$.

For the inverse class- $F$ PA with maximally flat waveforms, the pair of current and voltage waveforms can also be expressed in two ways,

$$
i(\tau)=I_{\mathrm{dc}} w_{\text {odd }}(\tau), \quad v(\tau)=V_{\mathrm{dc}} w_{\text {even }}^{*}(\tau)
$$

or

$$
i(\tau)=I_{\mathrm{dc}} w_{\mathrm{odd}}^{*}(\tau), \quad v(\tau)=V_{\mathrm{dc}} w_{\text {even }}(\tau),
$$

where $I_{\mathrm{dc}}>0$ and $V_{\mathrm{dc}}>0$.

As an example, a pair $\left(w_{\text {even }}^{*}, w_{\text {odd }}\right)$ for the case $N=9$, is presented in Figure 7.

The efficiency of class- $F$ and inverse class- $F$ PA via current and voltage waveform parameters $\gamma_{I}$ and $\gamma_{V}$ can be expressed as $\eta=\gamma_{I} \gamma_{V} / 2$ (see e.g., [2]), which according to our odd-even waveform notation leads to

$$
\eta=\frac{\gamma_{\text {even }} \gamma_{\text {odd }}}{2}
$$

From (30), (47), and (66) it follows that the efficiency of class$F$ PA (inverse class- $F$ PA) with maximally flat waveforms can be expressed as an explicit function of number of even

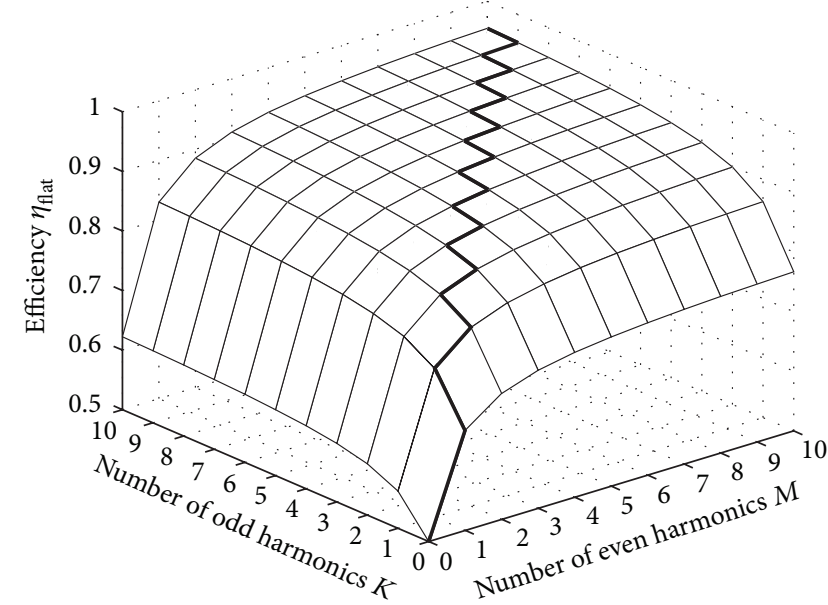

FIgURE 8: Efficiency of class- $F$ and inverse class- $F$ PA with maximally flat waveforms.

harmonics $M$ in voltage (current) waveform and number of higher odd harmonics $K$ in another waveform:

$$
\eta_{\text {flat }}=\frac{((2 M) ! !)^{2}}{(2 M-1) ! !(2 M+1) ! !} \cdot \frac{((2 K+1) ! !)^{2}}{(2 K) ! !(2 K+2) ! !} .
$$

As we pointed out earlier, the waveform parameters $\gamma_{\text {even }}$ and $\gamma_{\text {odd }}$ increase with number of harmonics and, therefore, this is the case with $\eta_{\text {flat }}$. The efficiency $\eta_{\text {flat }}$, as a function of $M$ and $K$, is plotted in Figure 8.

In what follows, we show how to choose $M$ and $K$, for the prescribed sum $M+K$, in order to ensure that efficiencies of class-F and inverse class-F with maximally flat waveforms are maximal.

Proposition 13. For a prescribed sum $M+K$, efficiency of class-F and inverse class-F with maximally flat waveforms (67) has maximum value if and only if

$$
0 \leq M-K \leq 1
$$

Proof. Let us first consider the quotient of efficiencies related to the pairs $(M, K)$ and $(M+1, K-1)$. According to (67), this quotient can be expressed as

$$
\begin{aligned}
& \frac{\eta_{\text {flat }}(M, K)}{\eta_{\text {flat }}(M+1, K-1)} \\
& \quad=\frac{(2 M+1)(2 M+3)}{(2 M+2)^{2}} \cdot \frac{(2 K+1)^{2}}{2 K(2 K+2)} .
\end{aligned}
$$

Expression (69) can be rewritten in the form

$$
\frac{\eta_{\text {flat }}(M, K)}{\eta_{\text {flat }}(M+1, K-1)}=1+\frac{(2 M+2)^{2}-(2 K+1)^{2}}{(2 M+2)^{2}\left(4 K^{2}+4 K\right)} \text {. }
$$

It is clear that efficiency $\eta_{\text {flat }}$ increases if and only if $(2 M+2)^{2}-$ $(2 K+1)^{2}>0$; that is, if and only if

$$
M-K>-\frac{1}{2} \text {. }
$$


Furthermore, let us consider the quotient of efficiencies related to the pairs $(M, K)$ and $(M-1, K+1)$. According to (67), this quotient can be expressed as

$$
\begin{aligned}
& \frac{\eta_{\text {flat }}(M, K)}{\eta_{\text {flat }}(M-1, K+1)} \\
& \quad=\frac{(2 M)^{2}}{(2 M-1)(2 M+1)} \cdot \frac{(2 K+2)(2 K+4)}{(2 K+3)^{2}} .
\end{aligned}
$$

Expression (72) can be rewritten in the form

$$
\frac{\eta_{\text {flat }}(M, K)}{\eta_{\text {flat }}(M-1, K+1)}=1-\frac{(2 M)^{2}-(2 K+3)^{2}}{\left(4 M^{2}-1\right)(2 K+3)^{2}} .
$$

It is clear that efficiency $\eta_{\text {flat }}$ increases if and only if $(2 M)^{2}-$ $(2 K+3)^{2}<0$; that is, if and only if

$$
M-K<\frac{3}{2}
$$

By combining (71) and (74), we finely obtain $-1 / 2<$ $M-K<3 / 2$, which clearly implies (68), since $M$ and $K$ are integers. This completes the proof.

Remark 14. From (68), if $M$ and $K$ are of the same parity (both are either even or odd), it follows that maximum value of $\eta_{\text {flat }}$ is achieved for $M=K$. If $M$ and $K$ are of different parity, then $M=K+1$ leads to the maximum value of $\eta_{\text {flat }}$. For maximally flat waveforms, the above consideration proves the fact that there is more benefit for efficiency in consecutive inclusion of harmonics alternatively in current and voltage waveforms than in inclusion of several harmonics in one waveform only (originally stated in [2]). As an illustration see bold zigzag line in Figure 8.

Remark 15. In the cases when $M=K$ or $M=K+1$ efficiency (67) reduces to a simple form expressed through single integer $N=1+M+K$ :

$$
\eta_{N, \text { flat }}=\frac{N}{N+1} \text {. }
$$

The cases of class- $F$ or inverse class- $F$ PA with $M=K$ or $M=K+1$ can be called "class- $F$ or inverse class- $F$ PA with $N$ harmonics."

The power-output capability of class- $F$ and inverse class$F$ PA can be expressed via current and voltage waveform parameters as $P_{\max }=\gamma_{I} \gamma_{V} /\left(2 \delta_{I} \delta_{V}\right)$ (e.g., see [2]). In our oddeven notation this expression can be rewritten as

$$
P_{\max }=\frac{\gamma_{\text {even }} \gamma_{\text {odd }}}{2 \delta_{\text {even }} \delta_{\text {odd }}} .
$$

Using (44) and (59), power-output capability of finite harmonic class- $F$ PA and inverse class- $F$ PA with maximally flat waveforms can be expressed as $P_{\max }=\gamma_{\text {odd }} / 8$. This result is in accordance with that obtained in [2]. Since $\gamma_{\text {odd }}$ can be expressed via $K$ (see (47)), it follows that $P_{\max }$ can be also expressed as an explicit function of $K$. When number of harmonics tends to infinity, according to (48), power-output capability tends to $P_{\max }=1 /(2 \pi)$.

\section{Conclusion}

In this paper, we consider general case of a problem of finding maximally flat waveforms with finite number of harmonics (maximally flat trigonometric polynomials). In Section 2 we prove that maximal degree of a degenerate critical point of waveforms with $N$ harmonics, not necessarily consecutive, is equal to $2 N-1$ (Lemma 1) and provide a closed form expressions for coefficients of such waveforms (Proposition 3).

In Section 3 we consider the so-called maximally flat even harmonic and maximally flat odd harmonic waveforms. We prove that these waveforms are nonnegative and have global minimum at maximally degenerate critical point (Propositions 6 and 10).

We provide closed form expressions for efficiency and power output capability of class- $F$ and inverse class- $F$ PA operations with maximally flat waveforms as functions of number of harmonics. We prove that maximal benefit in the increasing efficiency of class- $F$ and inverse class- $F$ PA operations with maximally flat waveforms can be achieved when harmonics are consecutively included in current and voltage waveforms. It is also shown that the efficiency of both class-F PA and inverse class- $F$ PA operation with maximally flat waveforms in the case of $N$ harmonics has a particular simple form $\eta_{N \text {,flat }}=N /(N+1)$.

\section{Acknowledgment}

This work is supported by the Serbian Ministry of Education, Science and Technology Development as a part of the Project TP32016.

\section{References}

[1] L. A. Novak and A. Juhas, "On the number of maxima of a sum of two commensurate harmonics: catastrophe curves," Tehnika, vol. 43, no. 1-2, pp. E7-E10, 1994.

[2] F. H. Raab, "Class-F power amplifiers with maximally flat waveforms," IEEE Transactions on Microwave Theory and Techniques, vol. 45, no. 11, pp. 2007-2012, 1997.

[3] A. Grebennikov and N. O. Sokal, Switchmode RF Power Amplifiers, Elsevier, Burlington, Mass, USA, 2007.

[4] A. Juhas, L. A. Novak, and S. Kostic, "Signals with flattened extrema in balance power analysis of HFHPTA: theory and applications," IEEE Transactions on Broadcasting, vol. 47, no. 1, pp. 38-45, 2001.

[5] A. Juhas and L. A. Novak, "Comments on "class-E, Class$\mathrm{C}$, and Class-F power amplifier based upon a finite number of harmonics"', IEEE Transactions on Microwave Theory and Techniques, vol. 57, no. 6, pp. 1623-1625, 2009.

[6] F. H. Raab, "Class-E, class-C, and class-F power amplifiers based upon a finite number of harmonics," IEEE Transactions on Microwave Theory and Techniques, vol. 49, no. 8, pp. 1462-1468, 2001.

[7] M. Roberg and Z. Popović, "Analysis of high-efficiency power amplifiers with arbitrary output harmonic terminations," IEEE Transactions on Microwave Theory and Techniques, vol. 59, no. 8, pp. 2037-2048, 2011. 


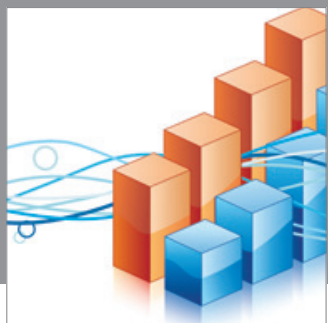

Advances in

Operations Research

mansans

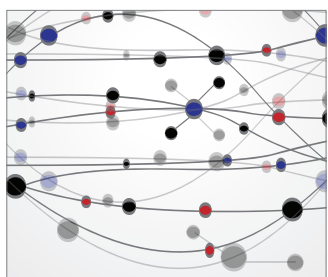

The Scientific World Journal
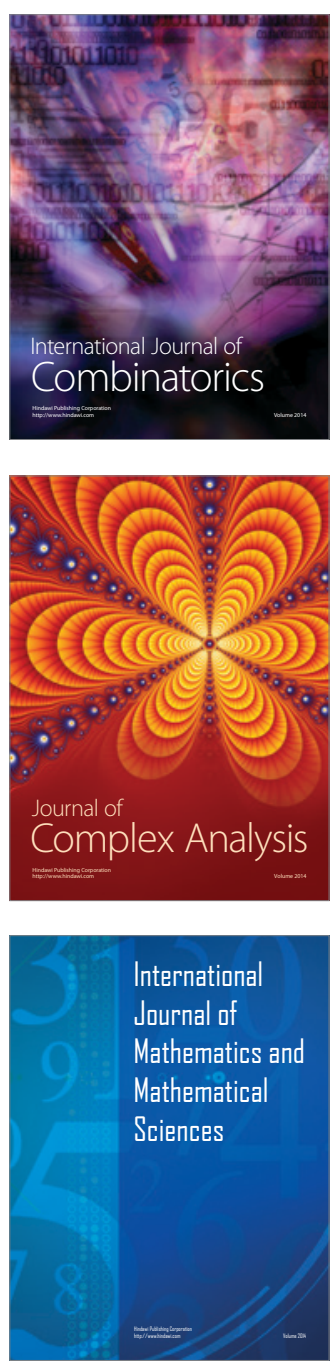
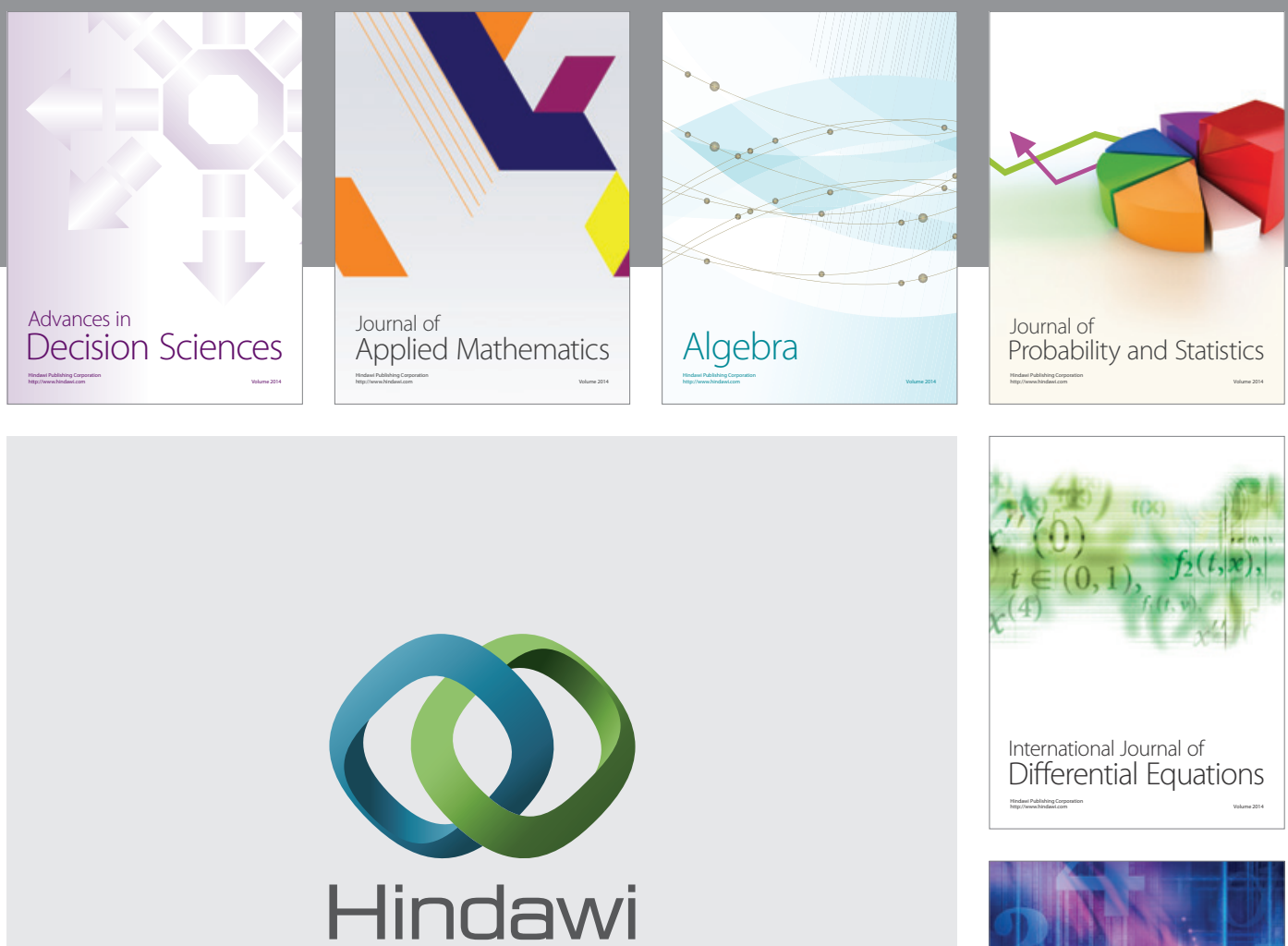

Submit your manuscripts at http://www.hindawi.com
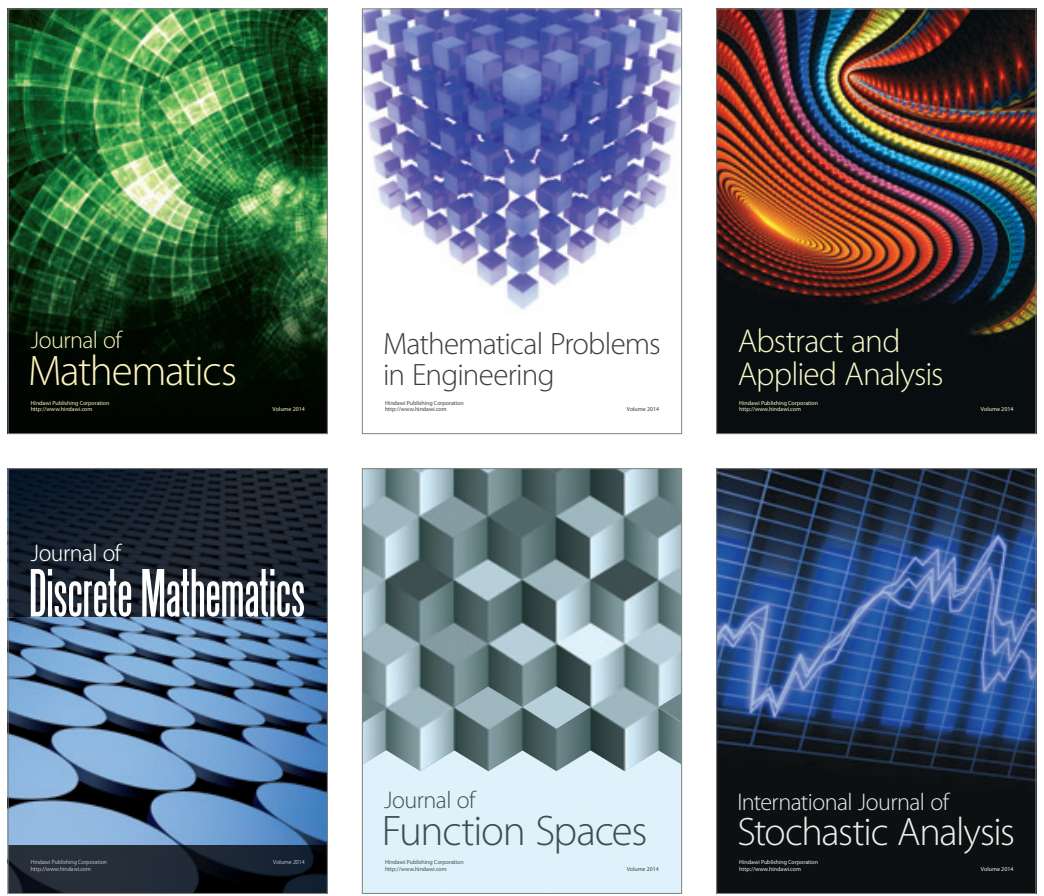

Journal of

Function Spaces

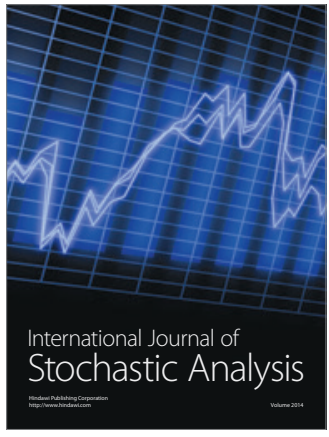

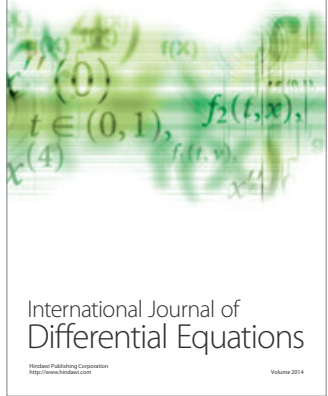
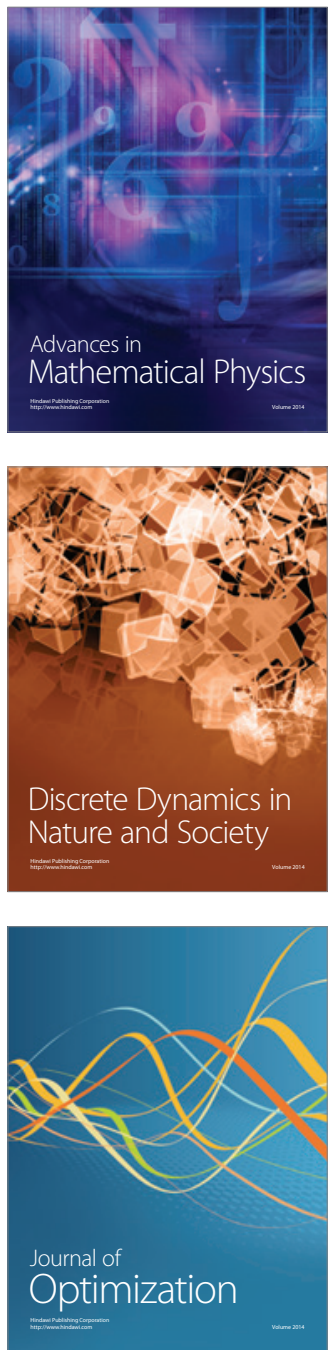